\title{
ON THE ROLE OF THE HEISENBERG GROUP IN HARMONIC ANALYSIS
}

\author{
BY ROGER HOWE
}

In this article I want to popularize the Heisenberg group, which is remarkably little known considering its ubiquity. I use the word ubiquity advisedly. To justify it, let me give a sample of the many apparently diverse topics where the Heisenberg group reveals itself as an important factor.

(1) Representation Theory of Nilpotent Lie Groups

(2) Foundations of Abelian Harmonic Analysis

(3) Moduli of Abelian Varieties

(4) Structure Theory of Finite Groups

(5) Theory of Partial Differential Equations

(6) Quantum Mechanics

(7) Homological Algebra

(8) Ergodic Theory

(9) Representation Theory of Reductive Algebraic groups

(10) Classical Invariant Theory

This list could easily be lengthened both by adding new topics and making these more specific, for sometimes the applications are multiple. In fact, one of the most important areas of application I have not mentioned at all, to avoid name-dropping. Why has an object with such wide application gone relatively unnoticed until recently? One can only speculate. One reason might be that the role of the Heisenberg group in many situations is relatively subtle. An investigator might be able to get what he wanted out of a situation while overlooking the extra structure imposed by the Heisenberg group, structure which might enable him to get much more. Such may have been the case with Hermann Weyl, one of the pioneers in introducing the Heisenberg group into Quantum Mechanics [Wy1]. Indeed, many physicists still call the Heisenberg group the "Weyl group". When Weyl wrote his book The classical groups, [Wy2] he overlooked the natural occurrence of the Heisenberg group, exploitation of which yields results which one feels Weyl would have liked very much. Again, I gather that an appreciation of the role of the Heisenberg group in rigidifying abelian varieties was an important aspect of Mumford's [Mm] fundamental contributions to their study. Another obstacle to the appreciation of the common underlying structure may have been the very diversity of the topics I listed above, for detection of its presence in one place need not suggest its presence elsewhere. Indeed, investigators in one field may very well never have been aware that the Heisenberg group had been found in some field not seemingly related to theirs. Another factor certainly contrib-

Received by the editors December 15, 1979.

AMS (MOS) subject classifications (1970). Primary 42-02, 35-02, 22-02.

Key words and phrases. Heisenberg group, Fourier transform, harmonic analysis, symbols. 
utory to its relative obscurity is that what I call "the Heisenberg group" is not in fact one object, but a collection of similar objects, rather like a functor, or a scheme in algebraic geometry, or even a combination of several overlapping functors. Thus one has to look with a certain pair of spectacles in order to see the topics on the list as being united via a single common phenomenon. But I think this is possible, and I want to give some examples of this point of view here. For people unacquainted with the Heisenberg group, this can be an introduction. Hopefully those already acquainted with it will also learn something.

These examples will be taken mostly from harmonic analysis. More particularly, except for the last, they are mostly an attempt to bridge the gap between abelian and nonabelian harmonic analysis by showing how various important results in abelian harmonic analysis may be enriched through an interpretation involving the Heisenberg group. Because of this choice of theme, the examples will not reveal the breadth of the range of applications of the Heisenberg group. But they do hint at more varied applications, and since they treat material that should be relatively familiar, they will perhaps be accessible to a reasonably wide audience. The topics are

(1) Plancherel $\Leftrightarrow$ Stone-von Neumann,

(2) distributional viewpoint towards Fourier Inversion and Poisson Summation,

(3) Bochner's Formula,
(4) Huyghens' Principle $\left(\mathrm{Sl}_{2}, \mathrm{O}_{p, q}\right)$ duality,

(5) symbols in the theory of pseudo-differential operators.

I take as my text for this discussion the article by E. M. Stein in Studies in harmonic analysis, J. Marshall Ash, ed., published by MAA (1976), entitled Harmonic analysis on $\mathbf{R}^{n}$. Before he gets to the more specialized recent developments, Stein lists a number of facts and formulas he considers basic to the subject of harmonic analysis on $\mathbf{R}^{n}$. These are

(a) the Fourier Inversion Theorem,

(b) the Schwartz Space,

(c) the Plancherel Theorem,

(d) the fact that Fourier transform interchanges $d / d x$ and multiplication by $2 \pi i x$, and translation by $y$ and multiplication by $e^{2 \pi i x \cdot y}$. (One might just say, that it interchanges convolution and multiplication.)

(e) that Fourier transform commutes with rotations, and more or less normalizes scalar dilations. (More generally, Fourier transform more or less normalizes the transformations coming from $G L_{n}$. If they are unitarized, it does do so.)

(f) that $\left(e^{-\pi x^{2}}\right)^{\wedge}=e^{-\pi x^{2}}$. Also that the Fourier transform on radial functions is given by a Hankel transform:

$$
\hat{f}(r)=(2 \pi r)^{-(n-2) / 2} \int_{0}^{\infty} f(s) J_{(n-2) / 2}(2 \pi r s) s^{n / 2} d s .
$$

Also Hecke's formula: $\left(P(x) e^{-\pi x \cdot x}\right)^{\wedge}=i^{k} P(x) e^{-\pi x \cdot x}$ if $P$ is a spherical harmonic on $\mathbf{R}^{n}$ of degree $k$; and the formula of 
Bochner, that $(P(x) f(r))^{\wedge}=P(x) T(f(r))$, where $T$ is the Hankel transform of degree $n / 2+k-1$.

These facts are presented by Stein, I think it is accurate to say, as interesting and important facts which happen to be true. I want to cast them in the role of clues pointing to a larger truth. I will try to do so without getting too doctrinaire or theological.

The usual point of view is that Fourier transform (herein denoted $\wedge$ ) takes (hard to understand) convolution operators to (easy to understand) multiplication operators, and this is certainly a vital function of $\wedge$. However, both multiplications and translations are special operators, and to get general operators we must combine them. Thus it might be worthwhile to take a more symmetrical point of view. This can be effected in a group theoretical context as follows. For $x, y \in \mathbf{R}^{n}$, define

$$
T_{x} f\left(x^{\prime}\right)=f\left(x^{\prime}-x\right), \quad M_{y} f\left(x^{\prime}\right)=e^{2 \pi i y \cdot x^{\prime}} f\left(x^{\prime}\right)
$$

where $y \cdot x^{\prime}$ is the usual inner product. Also for $x \in \mathbf{T}$, consider the scalar operators, $S_{z} f\left(x^{\prime}\right)=z f\left(x^{\prime}\right)$. Then the set $\left\{M_{y} T_{x} S_{z}: y \in \mathbf{R}^{n}, x \in \mathbf{R}^{n}, z \in \mathbf{T}\right\}$ is in fact a group of unitary operators on $L^{2}(\mathbf{R})$. Somewhat more abstractly, if we consider the set $H=\mathbf{R}^{n} \times \mathbf{R}^{n} \times \mathbf{T}$, and define on it a law of combination

$$
\left(y_{1}, x_{1}, z_{1}\right)\left(y_{2}, x_{2}, z_{2}\right)=\left(y_{1}+y_{2}, x_{1}+x_{2}, e^{-2 \pi i y_{2} \cdot x_{1}} z_{1} z_{2}\right)
$$

then $H$ becomes a two step nilpotent group we will call the (reduced) Heisenberg group of order $n$. Then the map

$$
\rho:(y, x, z) \rightarrow M_{y} T_{x} S_{z}
$$

is a faithful unitary representation of $H$. It is very easy to see that $\rho$ is irreducible. Since the $M_{y}$ 's separate points, any operator commuting with them is a multiplication [L1]. Since the $T_{x}$ 's act transitively, this multiplication operator must be constant, i.e., scalar.

The basic formulas for Fourier transform say that $\wedge$ normalizes $\rho(H)$. More precisely, let us define an automorphism $r$ of $H$ by the rule

$$
r(y, x, z)=\left(-x, y, e^{2 \pi i x \cdot y} z\right) .
$$

Then it is immediate to verify that

$$
\wedge \rho(h)=\rho(r(h))^{\wedge}
$$

That is to say, $\wedge$ intertwines the two representations $\rho$ and $\rho \circ r$ of $H$ with each other. When you have an intertwining operator between irreducible representations, it has to be terrible not to be unitary. In particular, if densely defined and closable, so if its adjoint is densely defined, it will be unitary (up to multiples). This is the general Schur's lemma [L2, p. 180]. It is relatively obvious that $\wedge$ has these weak properties, so it is unitary up to multiples. Thus computing one Fourier transform, any one, serves to show that in its standard form,

$$
\hat{f}(y)=\int_{\mathbf{R}^{n}} f(x) e^{-2 \pi i x \cdot y} d x,
$$

$\wedge$ is unitary. 
Fourier inversion can be dealt with similarly. If we repeat $\wedge$ twice we know $\wedge \wedge \rho(h)=\rho\left(r^{2}(h)\right)^{\wedge \wedge}$, where $r^{2}(y, x, z)=(-y,-x, z)$. It is trivial to check that $\omega(-1)$, defined by $\omega(-1) f(x)=f(-x)$ accomplishes the same intertwining. Thus by irreducibility of $\rho, \wedge^{2}=\omega(-1)$ up to multiples, and again a single computation suffices to establish that the normalization is correct. With regard to these computations, let us note they are both accomplished by the single formula, listed by Stein (see (1)(f)).

$$
\left(e^{-\pi x \cdot x}\right)^{\wedge}=e^{-\pi x \cdot x}
$$

For the proof of this, Stein says you make an obvious reduction to the one dimensional case, where it follows by a contour integral argument. A less sophisticated procedure in the one dimensional case is to observe that $e^{-\pi x^{2}}$ solves the differential equation

$$
(d / d x+2 \pi x) f=0 .
$$

Since this equation is invariant under Fourier transform, we see $e^{-\pi x^{2}}$ must be its own Fourier transform up to multiples. Thus the whole business is reduced to the famous formula

$$
\int_{-\infty}^{\infty} e^{-\pi x^{2}} d x=1
$$

The significance of this proof of (8) will be discussed later.

Thus simply the observation that the reduced Heisenberg group exists virtually suffices to establish the basic Plancherel and Fourier Inversion formulas. Reciprocally, the Plancherel Theorem serves to prove and strengthen a celebrated fact about the Heisenberg group, the Stone-von Neumann Theorem, which lies at the foundation of quantum mechanics. Thus the Plancherel and Stone-von Neumann Theorems are revealed as essentially equivalent. We proceed to detail this, bringing in the Schwartz space along the way.

When you have a unitary representation $\sigma$ of a Lie group $G$, it is natural to differentiate it and integrate it, and one has a notion of smooth vectors. Let $\$$ denote the Lie algebra of $H$. Let $e: \mathfrak{G} \rightarrow H$ be the exponential map. We can

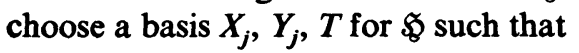

$$
e\left(\sum y_{j} Y_{j}+\sum x_{i} X_{i}+t T\right)=\left(y, x, e^{2 \pi i(t-y \cdot x / 2)}\right)
$$

where $y=\left(y_{1}, \ldots, y_{n}\right), x=\left(x_{1}, \ldots, x_{n}\right)$. Then we find easily that

$$
\rho\left(X_{j}\right)=-\partial / \partial x_{j}, \quad \rho\left(Y_{j}\right)=2 \pi i x_{j}, \quad \rho(T)=2 \pi i .
$$

The equations (12) are significant in several regards. For one thing, we can compute that

$$
\left[Y_{j}, X_{i}\right]=Y_{j} X_{i}-X_{i} Y_{j}=2 \pi i \delta_{i j}
$$

The equations (13) are the celebrated canonical commutation relations of Heisenberg. The also famous Stone-von Neumann Theorem asserts that under mild assumptions the operators of (12) are, up to unitary equivalence, the only realization of the canonical commutation relations. We will formulate the group theoretical version of this result. This is essentially the 
formulation of von Neumann [vN]. It is an interesting historical note that Hermann Weyl realized very early [Wy1] that the canonical commutation relations were the structural equations for a Lie algebra, and that there was an essentially equivalent formulation of them in terms of the corresponding Lie group. For this reason, among physicists, the group $H$ is often called the Weyl group. This terminology would obviously cause too much confusion among mathematicians, and also probably exemplifies some of the differences in psychology between mathematicians and physicists.

In any case, we have the following result.

TheOREM (STONE-vON NeUMANN). The representation $\rho$ of $H$ is, up to unitary equivalence, the only irreducible unitary representation of $H$ such that

$$
\rho((0,0, z))=z I .
$$

(To express (14), we say the central character of $\rho$ is the identity.)

Just as introducing the Heisenberg group facilitated proof of the Plancherel Theorem, the flow can be reversed, and the Plancherel Theorem used to prove the Stone-von Neumann Theorem. This comes about simply by integrating $\rho$ in a reasonably explicit way. The argument comes from I. Segal [Sg], and is almost to be found in von Neumann's original (1931) paper [vN]. A great virtue of it is that it proves a meaningfully stronger result than the one we have just stated.

Consider $f \in L^{1}(G)$. One defines $\rho(f)$ in the usual way:

$$
\rho(f)=\int_{H} f(g) \rho(g) d g .
$$

Defining left translation in the usual way,

$$
L_{h}(f)\left(h^{\prime}\right)=f\left(h^{-1} h^{\prime}\right)
$$

we find from (15) the relation

$$
\rho\left(L_{z} f\right)=z \rho(f) \text { for } z \in \mathbf{T} \subseteq H .
$$

Thus, let us define

$$
{ }^{\circ} f=\int_{\mathbf{T}} z^{-1} L_{z} f d z
$$

Then it is easy to see that

$$
\text { (a) } \rho\left({ }^{\circ} f\right)=\rho(f), \quad \text { (b) } L_{z}\left({ }^{\circ} f\right)=z\left({ }^{\circ} f\right) \text {. }
$$

The subspace $L^{1}(H, e)$ of $L^{1}$ consisting of $f$ satisfying (19)(b) is a two-sided convolution ideal in $L^{1}$ and $f \rightarrow^{\circ} f$ is a projection of $L^{1}(H)$ onto $L^{1}(H, e)$. Equation (19)(a) says $\rho$ factors through this projection. Hence in computing (15) it will suffice to consider only $f \in L^{1}(H, e)$. Such $f$ are determined by their restrictions $r(f)$ to the set $(y, x, 1) \subseteq H$. Let $\mathcal{S}\left(\mathbf{R}^{n}\right)$ be the Schwartz space of rapidly decreasing smooth functions on $\mathbf{R}^{n}[\mathbf{L 2}]$, [T]. Take $\phi \in \mathcal{S}\left(\mathbf{R}^{n}\right)$ 
and compute

$$
\begin{aligned}
\rho(f)(\phi)\left(x^{\prime}\right) & =\int_{\mathbf{R}^{n} \oplus \mathbf{R}^{n}} r(f)(y, x) M_{y}\left(T_{x} \phi\right)\left(x^{\prime}\right) d y d x \\
& =\int_{\mathbf{R}^{n} \oplus \mathbf{R}^{n}} r(f)(y, x) e^{2 \pi i y \cdot x^{\prime}} \phi\left(x^{\prime}-x\right) d y d x \\
& =\int_{\mathbf{R}^{n}} r(f)^{\wedge_{1}^{-1}}\left(x^{\prime}, x\right) \phi\left(x^{\prime}-x\right) d x
\end{aligned}
$$

(where $\wedge_{\mathbf{l}}^{-1}$ indicates inverse Fourier transform in the first variable)

$$
\begin{aligned}
& =\int_{\mathbf{R}^{n}} r(f)^{\wedge^{-1}}\left(x^{\prime}, x^{\prime}-x\right) \phi(x) d x \\
& =\int_{\mathbf{R}^{n}} \kappa_{f}\left(x^{\prime}, x\right) \phi(x) d x
\end{aligned}
$$

where

$$
\kappa_{f}\left(x^{\prime}, x\right)=r(f)^{\wedge_{1}^{-1}}\left(x^{\prime}, x^{\prime}-x\right)
$$

Segal calls formula (21) the Weyl transform. Thus $\rho(f)$ is an integral kernel operator. We recall that the integral operators with square integrable kernel form the algebra of Hilbert-Schmidt operators $\mathcal{H} . \mathcal{S}$. Also, we should note that there is a notion of smooth operator just as well as smooth vector. $\mathbf{A}$ smooth operator $T$ is one such that $\rho\left(u_{1}\right) T \rho\left(u_{2}\right)$ is bounded for all $u_{1}, u_{2}$ in the universal enveloping algebra of $H$. It is easy to see from formulas (12) that the smooth operators for $\rho$ are just the integral kernel operators with Schwartz functions as kernels. These clearly arise as Weyl transforms from $\mathcal{S}(H, e)$, the functions $f$ in $L^{1}(H, e)$ with $r(f)$ in $\mathcal{S}\left(\mathbf{R}^{2 n}\right)$. From our acquaintance with the Fourier transform, we may use formula (21) to conclude:

TheOREM (SEGAL). The representation map $\rho$ from $L^{1}(H, e)$ to bounded operators on $L^{2}\left(\mathbf{R}^{n}\right)$ extends to an isometric isomorphism from $L^{2}(H, e)$ to $\mathcal{H C} . \mathcal{S}$., and restricts to a topological isomorphism from $\mathfrak{S}(H, e)$ to the smooth operators for $\rho$.

This result makes it plausible that $\rho$ is unique, for there is in some sense nothing left from $L^{1}(H, e)$ out of which to make another representation. Indeed a few exercises in the yoga of matrix coefficients suffice to derive the Stone-von Neumann Theorem from this one.

The fact that $\rho$ extends from $L^{1}$ to $L^{2}$ means that $\rho$ is what is called a square-integrable representation. It is increasingly appreciated these days that square-integrability is an important property of representations.

We may combine the above results to obtain

TheOREM (STONE-VON NeUMANN-SEgal). Up to unitary equivalence there is a unique representation $\rho$ of $\boldsymbol{H}$ with the identity on $\mathrm{T}$ as central character, and this representation is square integrable.

In the light of recent results in representation theory, in particular those of Moore and Wolf [MW] on square-integrable representations, we can say that 
square-integrability is a natural thing to include in the description of $\rho$ because

THEOREM (MOORE-WoLf). An irreducible representation $\sigma$ of a nilpotent Lie group $N$ is square-integrable (modulo the center of $N$ ) if and only if it is the unique irreducible representation of $N$ with its central character.

From the uniqueness and square-integrability we can conclude immediately that $\rho$ is isometric from $L^{2}(H, e)$ to $\mathcal{H} . \delta$. Reading formula (21) backwards, we conclude that Fourier transform is isometric. Thus we conclude:

The Stone-von Neumann-Segal and the Plancherel Theorems are equivalent theorems.

Another aspect of the formulas (12) is that they imply immediately that the space of smooth vectors of $\rho$ is the Schwartz space $\mathcal{S}\left(\mathbf{R}^{n}\right)$. If we combine this with the Stone-von Neumann Theorem, we see that the Schwartz space is characterized abstractly as the smooth subspace $\rho^{\infty}$ of a certain irreducible representation $\rho$ of $\boldsymbol{H}$. Thus all questions of smoothness, growth, etc., of tempered distributions on $\mathbf{R}^{n}$ could in principle be formulated purely in terms of the structure of $\rho$. Although this procedure would of ten be counterproductive, it can be occasionally useful and is something to keep in mind. We will give an example as our last topic below.

Although $\boldsymbol{H}$ has only one representation with central character the identity up to unitary equivalence, there are many rather different looking ways of realizing this representation. This rather Hinduish multiplicity-in-one is characteristic of the theory of $H$ and adds greatly to its richness. We have already seen that the theory of the Fourier transform can be viewed as studying the intertwining between two realizations of $\rho$. The study of the intertwining between all realizations of $\rho$ leads in many directions, of which we can mention only a few.

Many realizations of $\rho$ are obtained by inducing. Let $A \subseteq H$ be any maximal abelian subgroup, and $\psi$ any unitary character of $\boldsymbol{A}$ restricting to the identity on $\mathbf{T}$. We may form the induced representation

$$
\operatorname{ind}_{A}^{H} \psi=\rho_{A, \psi}
$$

defined by left translation

$$
\rho_{A, \psi}(h) f\left(h^{\prime}\right)=f\left(h^{-1} h^{\prime}\right)
$$

on the space of functions $f$ such that

$$
f\left(h^{\prime} a\right)=\psi(a)^{-1} f\left(h^{\prime}\right), \quad \int_{H / A}|f|^{2} d h<\infty .
$$

It is clear that the central character of $\rho_{A, \psi}$ is the identity, and since $A$ is specified as maximal abelian, it is not too hard to show, for example via the general results of Mackey that

THEOREM. $\rho_{A, \psi}$ is irreducible, hence a realization of the unique irreducible representation $\rho$ of $H$ with the identity central character.

For example, if $A=\{(y, 0, z)\}$ and $\psi(y, 0, z)=z$, then we just get the original action of $H$ defined by formulas (2). In this case, since if $X=$ 
$\{(0, x, 1)\} \subseteq H$, we have $H=A \cdot X$, we can identify a function in the space of $\rho_{A, \psi}$ with a function on $X \simeq \mathbf{R}^{n}$, and then the action is just that given in (2). This is called the Schrödinger model of $\rho$. Since $H$ has a lot of symmetry, this gives a lot of realizations of $\rho$. We will see further examples below.

Corresponding to the realizations just described there is a distributional version of Frobenius reciprocity. Let $\rho^{\infty *}$ be the dual space of the smooth vectors $\rho^{\infty}$ of $\rho$. In the Schrödinger model, $\rho^{\infty *}$ is of course just the space of tempered distributions. On $\rho^{\infty *}$ one has the representation contragredient to the restriction of $\rho$ to $\rho^{\infty}$.

THEOREM. For maximal abelian $A \subseteq H$ and unitary character $\psi$ of $A$ restricting to the identity there is a linear functional $\mathcal{L}(A, \psi)$, unique up to multiples, such that

$$
\rho^{\infty *}(a) \mathcal{L}(A, \psi)=\psi(a)^{-1} \mathcal{Q}(A, \psi), \quad a \in A .
$$

That is,

$$
\mathcal{Q}(A, \psi)(\rho(a)(v))=\psi(a) \mathcal{L}(A, \psi)(v), \quad a \in A, v \in \rho^{\infty} .
$$

In the realization $\rho_{A, \psi}$ of $\rho$, the functional $\mathcal{2}(A, \psi)$ is just evaluation at the identity in $H$.

This theorem is probably due more to Cartier $[\mathbf{C r}]$ than anyone else.

Let us consider some concrete instances of this theorem. Let $A=$ $\{(y, 0, z)\}$ as before and $B=\{(0, x, z)\}$. Then if $\psi_{y}(y, 0, z)=z$ and $\psi_{x}(0, x, z)=z$, we can see in the Schrödinger model that $\mathcal{L}\left(A, \psi_{y}\right)=\delta_{0}$ is the Dirac delta at the origin in $\mathbf{R}^{n}$, and $\mathcal{Q}\left(B, \psi_{x}\right)=d x$ is Lebesgue measure on $\mathbf{R}^{n}$. These particular results may be proved in a down-to-earth fashion by the following advanced calculus lemmas.

LEMMA. If $f \in \mathcal{S}\left(\mathbf{R}^{n}\right)$ and $f(0)=0$, then we may write

$$
f=\sum_{i=1}^{n} x_{i} f_{i}
$$

with $f_{i} \in \mathcal{S}\left(\mathbf{R}^{n}\right)$.

LEMMA. If $f \in \mathcal{S}\left(\mathbf{R}^{n}\right)$ and $\int_{\mathbf{R}^{n}} f d x=0$, then we may write

$$
f=\sum_{i=1}^{n} \frac{\partial f}{\partial x_{i}}
$$

with $f_{i} \in \mathcal{S}\left(\mathbf{R}^{n}\right)$.

Of course these lemmas are just Fourier transforms of one another, but we want to take the opposite point of view. Suppose we have the general result on $\mathcal{2}(A, \psi)$, and let $\alpha$ be an automorphism of $H$ which acts trivially on T. By the uniqueness of $\rho$ we know from general considerations that there is a unitary operator $\omega(\alpha)$, defined up to scalar multiples, such that

$$
\omega(\alpha) \rho(h) \omega(\alpha)^{-1}=\rho(\alpha(h)) .
$$

If $A$ is a maximal abelian subgroup of $H$, then so is $\alpha(A)$. Let $\alpha^{*}(\psi)$ be the character on $\alpha(A)$ defined by

$$
\alpha^{*}(\psi)(\alpha(a))=\psi(a)
$$


It is clear that $\omega(\alpha)$ preserves $\rho^{\infty}$ since this is defined in terms of the action of $H$. Therefore we can define $\omega^{\infty *}(\alpha)$ acting on $\rho^{\infty *}$ in the usual manner:

$$
\omega^{* \infty}(\alpha)(\lambda)(v)=\lambda\left(\omega\left(\alpha^{-1}\right) v\right)
$$

Putting these formulas together, we find that

$$
\omega^{* \infty}(\alpha) \mathcal{2}(A, \psi)=\mathcal{2}\left(\alpha(A), \alpha^{*}(\psi)\right) \text {. }
$$

It is to be understood that (31) is an equation in projective space. If we apply equation (31) to $A=\{(y, 0, z)\}$ and $\alpha=r$ as in formula (5), then we find by formula (6) and our lemmas that Lebesgue measure is the Fourier transform of the Dirac delta, which is simply the Plancherel Theorem from the distributional point of view.

A nice feature of this approach to Fourier transform is that it puts it on the same footing as the Poisson Summation Formula. Thus let $\Gamma=\{(a, b, z)\}$ where $a, b \in \mathbf{Z}^{n}$. It is easily checked that $\Gamma$ is a maximal abelian subgroup of $H$. Let $\psi$ be the character of $\Gamma$ such that $\psi(a, 0,1)=1=\psi(0, b, 1)$. It is easy to see that the automorphism $r$ of formula (5) fixes $\Gamma$ and $\psi$. In the Schrödinger model we conclude that $2(\Gamma, \psi)$ is an eigendistribution for Fourier transform. It is easy to check that Haar (counting) measure on $\mathbf{Z}^{\boldsymbol{n}}$ is invariant under $\rho(a, 0,1)$ and $\rho(0, b, 1)$, hence must be $2(\Gamma, \psi)$. (A direct proof that it is the unique distribution with these invariance properties is another advanced calculus exercise.) Thus counting measure on $\mathbf{Z}^{n}$ is, up to multiples, invariant under Fourier transform. Again the formula (8) allows us to fix the multiple as 1. Thus we have the Poisson Summation Formula.

The gentle reader will hopefully also have been reminded by the above discussion of our argument for formula (8). Indeed, formula (8) is retated to another class of realizations of $\rho$, not induced in the usual sense, but sometimes called holomorphically induced, and also called Fock models or complex wave models. Study of the Fock model leads into the study of $\theta$-functions, Toeplitz operators and other topics. Indeed, study of the $2(A, \psi)$ leads in many directions. They are a central concern in the theory of the Heisenberg group.

We have now discussed most of the topics on Stein's list. There remains the relation of Fourier transform to $G L_{n}$ and Bochner's and Hecke's formulas. These topics lead deeper into the theory, to the construction of a certain unitary representation with many names. I shall call it the oscillator representation because of its connection with the quantum harmonic oscillator and its analogy with the spin representation. Once having gone so far we will find other topics are also approachable. We will discuss a very classical oneHuyghens' Principle for the wave equation.

Of course $G L_{n}$ is the group of automorphisms $\mathbf{R}^{n}$. We see further that if $g \in G L_{n}$, then

$$
\alpha(g):(y, x, z) \rightarrow\left(g^{t^{-1}}(y), g(x), z\right),
$$

where $g^{t^{-1}}$ is the inverse transpose of $g$, defines an automorphism of $H$. We more or less began this discussion by observing that Fourier transform also defined an automorphism $r$ of $H$. It can be observed from (5) and (32) that $r$ 
normalizes $\alpha\left(G L_{n}\right)$. Precisely

$$
r \alpha(g) r^{-1}=\alpha\left(g^{t^{-1}}\right) .
$$

This has the following operator theoretic consequence.

For $g \in G L_{n}$, we define $\omega(g)$ on $L^{2}\left(R^{n}\right)$ by

$$
\omega(g) f(x)=|\operatorname{det} g|^{-1 / 2} f\left(g^{-1}(x)\right) .
$$

Then $\omega(g)$ is unitary and satisfies (28). We conclude from (33) and (6) that, up to a multiple which one again checks to be 1 ,

$$
\wedge_{\omega}(g)=\omega\left(g^{t^{-1}}\right)^{\wedge} \text {. }
$$

Formula (33') is of course very easy to verify directly.

Thus (33') arises because $\wedge$ normalizes $\alpha\left(G L_{n}\right)$ in the automorphisms of the Heisenberg group. At this point it is natural to ask for all the automorphisms of $\boldsymbol{H}$ acting trivially on T. This is most easily attacked by transferring the problem to the Lie algebra $\mathfrak{S}$ of $H$, as described in (11) and (12). Put $W=\operatorname{span}\left\{X_{j}, Y_{j}\right\}$, so that

$$
\mathfrak{S}=W \oplus \mathbf{R} .
$$

First there are inner automorphisms. These are easily checked to act simply transitively on the linear complements to $\mathbf{R}$ in $\mathfrak{G}$. Therefore we may identify the outer automorphism group of $\mathfrak{g}$ with the group of automorphisms fixing $W$. From formulas (12) it is evident that Lie bracket on $\mathfrak{G}$ induces a symplectic form $\langle$,$\rangle on W$. Evidently any automorphism of $\mathfrak{S}$ fixing $\mathbf{R}$ pointwise and leaving $W$ invariant must preserve $\langle$,$\rangle . And from (12) one$ can also see that conversely any isometry of $\langle$,$\rangle on W$ extends in obvious fashion to an automorphism of $\mathfrak{g}$. We will denote the isometry group of $\langle$, by $\operatorname{Sp}(W,\langle\rangle)=,\mathrm{Sp}$ and will consider it as a group of automorphisms of $H$. If we note that $W=\mathbf{R}^{n} \oplus \mathbf{R}^{n}$, where the first factor is the span of the $Y$ 's and the second the span of the $X$ 's, then $\alpha\left(G L_{n}\right)$ is the subgroup of Sp preserving this decomposition, and the transformation $r$ of (5) is precisely the nontrivial element of the normalizer of $\alpha\left(G L_{n}\right)$ in Sp.

As we noted above we get for each $g \in \mathrm{Sp}$ a unitary operator $\omega(g)$ defined up to multiples and satisfying (28). We thus get a well-defined action $\omega$ of Sp on the projective space $\mathbf{P} L^{2}\left(\mathbf{R}^{n}\right)$. Such an action is called a projective unitary representation. An ordinary, linear, unitary representation gives rise to a projective representation by just looking at the action on lines. A natural question is the extent to which the reverse is true, and this has been considered in a general context by Mackey [Ma]. In the present situation one knows [Sh], [Wi].

Theorem (Shale-WeiL). Let $\mathrm{S} p$ be the 2-fold cover of Sp. Let $\tilde{\mathbf{g}} \rightarrow g$ be the projection map. Then there is a unitary representation $\omega$ of $\tilde{\mathrm{S} p}$ on $L^{2}$ such that

$$
\omega(\tilde{g}) \rho(h) \omega(\tilde{g})^{-1}=\rho(g(h)) .
$$

We will call $\omega$ the oscillator representation.

We will use this theorem to abuse notation and not distinguish between $\omega$ as a projective representation of $\mathrm{Sp}$ and as a linear representation of $\tilde{\mathrm{Sp}}$. 
We should describe $\omega$ a little bit. We have already described $\omega\left(\alpha\left(G L_{n}\right)\right)$ and $\omega(r)$. Let $N$ be the subgroup of Sp which leaves the $Y_{j}$ 's fixed. Then $N$ is isomorphic to the space of symmetric $n \times n$ matrices in such a way that $\omega(N)$ consists of multiplications by functions

$$
e^{\pi i Q(x, x)}, \quad Q \text { symmetric } n \times n .
$$

Since $\alpha\left(G l_{n}\right), N$ and $r$ generate Sp, this in principle describes all $\omega(g)$, and we will leave the matter there. It is a property of $\omega$ not to be transparently describable in any realization. However, letting $\mathfrak{s p}$ denote the Lie algebra of $\mathrm{Sp}$, we can easily describe $\omega(\mathfrak{S p})$. It consists of the differential operators of "total order" 2 , namely

$$
\pi i x_{i} x_{j}, \quad \frac{i}{4 \pi} \frac{\partial^{2}}{\partial x_{i} \partial x_{j}} \text { and } \frac{1}{2}\left(x_{i} \frac{\partial}{\partial x_{j}}+\frac{\partial}{\partial x_{j}} x_{i}\right)=x_{i} \frac{\partial}{\partial x_{j}}+\frac{\delta_{i j}}{2} .
$$

It is the necessity of adding $\frac{1}{2}$ trace to the linear vector fields in order to unitarize the action of $G L_{n}$ that leads to the order 2 obstruction to linearizing $\omega$ as a representation of $\mathrm{Sp}$ (as opposed to $\tilde{\mathrm{S}} \mathrm{p}$ ).

The oscillator representation has a number of remarkable properties. We are going to give an example of one of the most remarkable, then show how it relates to the topics mentioned above. Fix an inner product (, ) on $\mathbf{R}^{n}$. For convenience say $n=p+q$ and

$$
(x, y)=x_{1} y_{1}+\cdots+x_{p} y_{p}-x_{p+1} y_{p+1} \cdots-x_{n} y_{n} .
$$

Let $O_{p, q}=O$ be the isometry group of (, ). Put

$$
\rho_{p, q}^{2}=\rho^{2}(x, x) \text {. }
$$

(We will no longer be referring to the representation $\rho$ of $H$, though, of course, it is there.) Also put

$$
\Delta_{p, q}=\Delta=\sum_{j=1}^{p} \frac{\partial^{2}}{\partial x_{j}^{2}}-\sum_{j=p+1}^{p+q} \frac{\partial^{2}}{\partial x_{j}^{2}} .
$$

Then $\rho^{2}$ and $\Delta$, as differential operators, obviously commute with $\omega(O)$. Also, $\rho^{2}$ and $\Delta$ belong to the Lie algebra $\omega(\mathfrak{s p})$. We compute

$$
\left[\Delta, \rho^{2}\right]=4 \tilde{E}=4\left(\sum_{j=1}^{p+q} x_{j} \frac{\partial}{\partial x_{j}}+\frac{p+q}{2}\right) .
$$

It may easily be verified that $\Delta, \rho^{2}$ and $\tilde{E}$ span a Lie algebra $\mathfrak{g}$, with commutation relations

$$
\begin{aligned}
{\left[\tilde{E}, i \pi \rho^{2}\right] } & =2 \pi i \rho^{2}=2\left(i \pi \rho^{2}\right), \\
{\left[\tilde{E}, \frac{i \Delta}{4 \pi}\right] } & =-\frac{i \Delta}{2 \pi}=-2\left(\frac{i \Delta}{4 \pi}\right), \\
{\left[i \pi \rho^{2}, \frac{i \Delta}{4 \pi}\right] } & =\tilde{E} .
\end{aligned}
$$

These will be recognized as the commutation relations of a standard basis for $\mathfrak{s i}_{2}$. It is easy to check further that $\mathfrak{g}^{\prime}$ is the full Lie subalgebra of $\mathfrak{g} \mathfrak{p}$ commuting with $\omega(O)$. Let $G^{\prime}$ be the subgroup of Sp generated by $g^{\prime}$. Then 
$G^{\prime}$ is the centralizer of $O$ in Sp, and in fact, the relation is mutual: $\left(O, G^{\prime}\right)$ is a pair of groups in $\mathrm{Sp}$, each of which is the centralizer of the other. We call such a pair a dual pair. Let $\tilde{O}, \tilde{G}^{\prime}$ be the inverse images of $O$ and $G^{\prime}$ in $\tilde{\text { Sp}}$. Then $\left(\tilde{O}, \tilde{G}^{\prime}\right)$ are a dual pair in $\tilde{S}$. Hence if we restrict $\omega$ to $\tilde{O}$ or $\tilde{G}^{\prime}$, each group gives us operators commuting with the other, and thus helps us to decompose the restriction of $\omega$ to the other group. It turns out that the situation in this regard is as nice as it could be [Hw1].

THEOREM D. The groups $\omega(\tilde{O})$ and $\omega\left(\tilde{G}^{\prime}\right)$ of operators generate each other's commutants, in the sense of von Neumann algebras. Thus

$$
\omega \mid \tilde{O} \times \tilde{G}^{\prime} \simeq \int_{\tilde{\tilde{G}}^{\prime}} \sigma \otimes \sigma^{\prime} d \omega\left(\sigma^{\prime}\right)
$$

where $\hat{\tilde{G}}^{\prime}$ is the unitary dual of $\tilde{G}^{\prime}$, and $\sigma \in \hat{\tilde{O}}$, and the map $\sigma^{\prime} \rightarrow \sigma$ is an injection almost everywhere with respect to the spectral measure $d \omega\left(\sigma^{\prime}\right)$.

We will consider this result in the cases $q=0$ and $q=1$. When $q=0$ we discover Bochner's and Hecke's formulas. When $q=1$ we discover Huyghens' principle. Before beginning the details we note two general properties of $\omega \mid \tilde{G}^{\prime}$.

(1) From the general considerations about $\omega$ it is easy to see that if $p+q$ is even, then $\tilde{G}^{\prime}$ is just $S l_{2}$, but if $p+q$ is odd, then $\tilde{G}^{\prime}$ is the nontrivial 2 -fold cover of $S l_{2}$, with the nontrivial element of the kernel of the covering map acting by minus the identity on $L^{2}(X)$.

(2) The generator of $\mathrm{SO}_{2}$ (or its cover) in $\tilde{G}^{\prime}$ is $(i / 2)\left(2 \pi \rho^{2}-\Delta^{2} / 2 \pi\right)$ which is just an indefinite Hermite operator. Hence $e^{(\pi i / 2)\left(2 \pi \rho^{2}-\Delta^{2} / 2 \pi\right)}$ is essentially just the Fourier transform "adapted to (, )" that is,

$$
{ }^{\wedge p, q}(f)(y)=\int_{\mathbf{R}^{p+q}} f(x) e^{-2 \pi i(x, y)} d x .
$$

In other words

$$
\begin{aligned}
\exp \left(\frac{\pi}{2} i\left(\frac{\pi \rho^{2}}{2}-\frac{\Delta^{2}}{4 \pi}\right)\right)=i^{(p-q) / 2 \wedge p, q}= & \pm \omega\left(\begin{array}{cc}
0 & 1 \\
-1 & 0
\end{array}\right) \\
& \text { where }\left(\begin{array}{cc}
0 & 1 \\
-1 & 0
\end{array}\right) \in S l_{2} \simeq G^{\prime} .
\end{aligned}
$$

If $q=0$, then $O_{p, 0}=O_{p}$ is compact, and by general considerations [L1] we know we can write $L^{2}\left(\mathbf{R}^{n}\right)=\bigoplus_{j=0}^{\infty} H_{j}$ where the $H_{j}$ are isotypic spaces for $O_{p}$. The duality theorem above then says that each $H_{j}$ is irreducible as an $O_{p} \times \tilde{G}^{\prime}$ module. Thus

$$
H_{j} \simeq \sigma_{j} \otimes \sigma_{j}^{\prime}
$$

where $\sigma_{j}$ is a finite-dimensional representation of $O_{p}$, and $\sigma_{j}^{\prime}$ is the corresponding representation of $\tilde{G}^{\prime}$. As we have mentioned above, the generator of a maximal compact subgroup $K$ of $\tilde{G}^{\prime}$ is $(i / 2)\left(2 \pi \rho^{2}-\Delta / 2 \pi\right)$. The Hermite operator $\rho^{2}-\Delta / 4 \pi^{2}$ is obviously nonnegative. Thus $\omega \mid K$ will have only one-sided spectrum. The representation of $\tilde{G}^{\prime}$ in which $K$ has one-sided spectrum are called holomorphic representations and are very well under- 
stood [L1]. This is what they look like. Put

$$
\begin{aligned}
\mathfrak{t} & =i\left(\pi \rho^{2}-\Delta / 4 \pi\right) \\
n^{+} & =\frac{\tilde{E}-\pi \rho^{2}-(\Delta / 4 \pi)}{2}=-\frac{1}{2} e^{\pi \rho^{2}}(\Delta / 4 \pi) e^{-\pi \rho^{2}} \\
n^{-} & =\frac{\tilde{E}+\pi \rho^{2}+(\Delta / 4 \pi)}{2}=\frac{1}{2} e^{-\pi \rho^{2}}(\Delta / 4 \pi) e^{\pi \rho^{2}} .
\end{aligned}
$$

Then from (42) we find

$$
\left[\mathfrak{f}, n^{+}\right]=2 i n^{+}, \quad\left[\mathfrak{f}, n^{-}\right]=-2 i n^{-}, \quad\left[n^{+}, n^{-}\right]=-i f .
$$

Any unitary representation of $\tilde{G}^{\prime}$ in which - if has positive spectrum contains a unique vector $v_{0}$ such that $n^{-} v_{0}=0$ and $\mathfrak{f} v_{0}=i l v_{0} / 2$ for some positive integer $l$. The vector $v_{0}$ is called the lowest weight vector for the representation, and $l / 2$ is the lowest weight. The space of the representation then has an orthogonal basis consisting of the vectors $v_{m}=n^{+m} v_{0}$. These are eigenvectors for $t$ with eigenvalues $i(l / 2+2 m)$. Denote by $D_{l / 2}$ the representation with lowest weight $l / 2$.

From this description and (46) we see that the kernel of $n^{-}$will contain exactly one copy of each representation of $O_{n}$ that occurs in $L^{2}\left(\mathbf{R}^{n}\right)$, and the irreducible $O_{n}$ modules in $\operatorname{ker} n^{-}$will just be the eigenspaces of $f$. From the second expression for $n^{-}$in (47) we see that ker $n^{-}$consists of functions of the form $\varphi e^{-\pi \rho^{2}}$ where $\Delta \varphi=0$, i.e., $\varphi$ is harmonic. Since $\varphi$ is harmonic it is analytic and we may expand it in a power series

$$
\varphi=\sum \varphi_{j}
$$

where $\varphi_{j}$ is a polynomial of degree $j$. Each $\varphi_{j}$ will of course also be harmonic. From the formulas (47) we can easily compute that

$$
\mathfrak{f}\left(\varphi e^{-\pi \rho^{2}}\right)=i(\tilde{E} \varphi) e^{-\pi \rho^{2}} .
$$

Hence $\varphi e^{-\pi \rho^{2}}$ is an eigenvector for $\mathfrak{t}$ if and only if $\varphi$ is a homogeneous polynomial. If $\varphi$ has degree $m$, then

$$
\mathfrak{f}\left(\varphi e^{-\pi \rho^{2}}\right)=i(m+p / 2) \varphi e^{-\pi \rho^{2}} .
$$

Let $\mathcal{K}_{m}$ be the harmonic polynomials of degree $m$. Since $\left(x_{1}+i x_{2}\right)^{m}$ is harmonic, we see $\mathcal{H}_{m} \neq\{0\}$ if $p>2$. If $p=1$, then $\mathcal{K}_{0}=\mathbf{C}, \mathcal{K}_{1}=\mathbf{C} x$, $\mathcal{K}_{i}=0$ for $i>2$.

A computation shows that the space of functions $\varphi f(r)$ is invariant by $n^{+}$if $\varphi$ is homogeneous and harmonic. We summarize the consequences of the above computations in the light of Theorem $D$. We restrict to $p>2$ for simplicity.

THEOREM. For $p>2$, we have $S\left(\mathbf{R}^{n}\right)=\sum_{m=0}^{\infty} \mathcal{H}_{m} \otimes G$ where $\mathcal{G} \subseteq \mathcal{S}$ are rotationally invariant functions and $\mathcal{K}_{m}$ is the space of harmonic polynomials of degree m. Each $\mathcal{H}_{m}$ is irreducible as $O_{p}$ module. Each space $\mathcal{H}_{m} \otimes g$ is 
irreducible under $\omega \mid O_{p} \times \tilde{G}^{\prime}$, and as $O_{p} \times \tilde{G}^{\prime}$ module has the form

$$
\mathcal{H}_{m} \otimes D_{m+p / 2}
$$

Thus we recover the theory of spherical harmonics with extra structure. Furthermore, the formulas of Hecke and Bochner are now clear. Indeed Hecke's formula is immediate since if $\varphi \in \mathcal{H}_{m}$, then $\varphi e^{-\pi \rho^{2}}$ is an eigenvector for $\mathfrak{f}$ of eigenvalue $m+p / 2$ and $e^{\pi i t / 2}=i^{p \wedge}$, as we remarked at the outset. As for Bochner's formula, the curious thing about it is not its specific form-that is what it is-but that restriction of $\wedge$ to $\mathcal{H C}_{m} \otimes g$ depends only on $m+p / 2$. From the viewpoint elaborated above, we can say that all the ingredients in Bochner's formula are defined in terms of $\tilde{G}^{\prime}$ alone, and therefore the form of the operator will depend only on which $\tilde{G}^{\prime}$ module is involved-and that, from (51) and (52) depends only on $m+p / 2$. This fact in itself can be predicted from the internal structure of the representation theory of $\tilde{G}^{\prime}$, and general considerations about $\omega$, but we won't go into that.

We now turn to the Lorentz case $q=1$. One can decompose $L^{2}\left(R^{p+1}\right)$ under $\omega \mid O_{p, 1} \times \tilde{G}^{\prime}$ explicitly as we did above when $q=0$, and this has been done by Strichartz [St] and by Rallis and Schiffman [RS]. Here, however, we will discuss a more special topic, Huyghens' Principle [Hw2].

The situation is this. One considers the wave equation

$$
\sum_{j=1}^{p} \frac{\partial^{2} \varphi}{\partial x_{j}^{2}}=\frac{\partial^{2} \varphi}{\partial t^{2}}
$$

One wants to solve the initial value problem: given $\varphi(x, 0)=\varphi_{0}(x)$ and $\partial \varphi(x, 0) / \partial t=\varphi_{0}^{\prime}(x)$, find $\varphi$ solving (53). Standard techniques involving the Fourier transform show there exist distributions $P_{1}$ and $P_{2}$ on $\mathbf{R}^{P+1}$ such that

$$
\varphi(x, t)=P_{1} * \varphi_{0}+P_{2} * \varphi_{0}^{\prime}
$$

where the convolution is in the $x$-variables only. The distributions $P_{i}$ are called propagators. They have been computed classically and the following results are known.

(a) $\varphi\left(x_{1}, t\right)$ depends only on the values of $\varphi_{0}(x)$ and $\varphi_{0}^{\prime}(x)$ for $\left\|x-x_{1}\right\|<$ $|t|$. In other words $P_{j}(, t)$ is supported in $\{x:\|x\|<|t|\}$. In other words $P_{j}$ is supported inside the light cone, i.e., in the set $C^{-}=\left\{(x, t), \Sigma x_{j}^{2}-t^{2}<0\right\}$.

(b) If $p$ is odd and $>1$, then $\varphi\left(x_{1}, t\right)$ depends only on the values of $\varphi_{0}(x)$ and $\varphi_{0}^{\prime}(x)$ (and their derivatives) for $\left\|x-x_{1}\right\|=|t|$. In other words, $P_{j}(, t)$ is supported on $\{x:\|x\|=|t|\}$. In other words, $P_{j}$ is supported on the light cone $C_{0}=\left\{(x, t): \Sigma x_{j}^{2}-t^{2}=0\right\}$.

The circumstances of (b), that signals are extinguished suddenly in odd (e.g., (3)) space dimensions is what is known as Huyghens' Principle today. We want to see how it comes out of the group theory.

We begin by observing that the wave equation (53) or more to the point, the initial value problem for the wave equation, has certain symmetries, and these symmetries are reflected in symmetries of $P_{1}$ and $P_{2}$. (Although the wave equation itself is invariant under the full Lorentz group $O_{n, 1}$ as well as dilations of space-time, the initial value problem has somewhat less symmetry since one must also worry about preserving the initial conditions.) Investigat- 
ing these symmetries, we find

$$
\begin{aligned}
& \omega(R) P_{j}=P_{j} \text { where } R \in O_{n} \text { is a rotation in the } x \text { 's. } \\
& \omega(s I) P_{1}=|s|^{(p+1) / 2} P_{1}, \omega(s I) P_{2}=|s|^{(p-1) / 2} \operatorname{sgn}(s) P_{2} \\
& \text { where } s I \text { is a dilation by } s \text { on all } \mathbf{R}^{p+1} \text {. } \\
& P_{j} \text { satisfies the wave equation. }
\end{aligned}
$$

Although (iii) might not usually be considered a symmetry of $P_{j}$, it is appropriate to so call it in our present context since the wave operator is just one of the infinitesimal generators of the $\tilde{G}^{\prime}$ commuting with $O_{n, 1}$.

The conditions (54) can be shown to characterize the $P_{j}$ up to multiples. Indeed, let $\wedge_{p, 1}=\wedge$ be the Fourier transform adapted to $\Sigma x_{j}^{2}-t^{2}$ on $\mathbf{R}^{p+1}$. Then we see from (54) that

$$
\begin{gathered}
\omega(R) \hat{P}_{j}=\hat{P}_{j} . \\
\omega(s I) \hat{P}_{1}=|s|^{-(p+1) / 2} \hat{P}_{1}, \omega(s I) \hat{P}_{2}=|s|^{-(p-1) / 2} \operatorname{sgn}(s) P_{2} . \\
\left(r^{2}-t^{2}\right) \hat{P}_{j}=0 .
\end{gathered}
$$

Equation (55)(iii) says $\hat{P}_{j}$ is supported on the light cone $C^{0}$. (Indeed, away from zero, it must factor through the restriction map to the light cone.) Then since $O_{p}$ and dilations together act transitively on $C^{0}$, we find, taking degrees of homogeneity into account, that

$$
\hat{P}_{1}=b_{1} t^{2} d \mu, \quad \hat{P}_{2}=b_{2} t d \mu
$$

where $d \mu$ is the unique $O_{p, 1}$ invariant measure on $C^{0}-\{0\}$.

Consider now the matter of Huyghens' Principle. Equations (54) say that $P_{j}$, aside from being $O_{n}$-invariant, is killed by the wave operator and is an eigenvector for the Euler operator $E$. Recalling formulas (42), we see this says $\boldsymbol{P}_{j}$ is a highest weight vector for the $\tilde{G}^{\prime}$ module it generates. We have seen Huyghens' Principle holds exactly when $P_{j}$ is supported on $C^{0}$. Since $C^{0}$ is just the locus of zeroes of $\Sigma x_{j}^{2}-t^{2}$, we know $P_{j}$ is supported on $C^{0}$ if and only if

$$
\left(\sum x_{k}^{2}-t^{2}\right)^{l} P_{j}=0
$$

for some $l$. But in the light of (54)(ii) and (iii) this is equivalent to saying $\boldsymbol{P}_{j}$ generates a finite-dimensional module for $\tilde{G}^{\prime}$. That is, we have the

CRITERION. Huyghens' Principle holds if and only if $\boldsymbol{P}_{j}$ generates a finitedimensional module for $\tilde{G}^{\prime}$.

As an immediate corollary we see that Huyghens' Principle must fail in odd space-time (even space) dimensions, because then, as we remarked above, $\tilde{G}^{\prime}$ is not $\mathrm{Sl}_{2}$ but the two-fold cover, which has no (faithful) finite-dimensional representations.

It is possible, however, for Huyghens' Principle to hold in even space-time dimensions. Taking Fourier transform, we see Huyghens' Principle will hold if and only if $\hat{P}_{j}$ generates a finite-dimensional $\tilde{G}^{\prime}$ representation of which it will then be the lowest weight vector. This is guaranteed by the following 
result, which is a distributional partial analogue to the $L^{2}$-duality theorem.

THEOREM D*. If $p+1$ is even, the distribution $Q(x, t) d \mu$, with $d \mu$ as in (56) and $Q$ a harmonic polynomial of degree $m$, generates the $\tilde{G}^{\prime}$ module $V_{m+(p-1) / 2}$ of dimension $m+(p-1) / 2$. The subspace of $\mathcal{S}^{*}\left(\mathbf{R}^{p+1}\right)$ transforming according to $V_{m+(p-1) / 2}$ under $\tilde{G}^{\prime}$ is finite dimensional and irreducible for the joint action of $O_{p, 1} \times \tilde{G}^{\prime}$ and isomorphic to the tensor product

$$
\mathcal{H}_{m} \otimes V_{m+(p-1) / 2}
$$

For $m=1,2$, we find the $\hat{P}_{j}$ generates a finite-dimensional $\tilde{G}^{\prime}$ module, since $t$ is harmonic, and

$$
t^{2}=\frac{p}{p-1}\left(t^{2}-\frac{1}{p} \sum_{j=1}^{p} x_{i}^{2}\right)-\frac{1}{p-1}\left(t^{2}-\sum_{j=1}^{p} x_{i}^{2}\right)
$$

where the first term is harmonic, and the product of the second term with $d \mu$ is zero. Thus Huyghens' Principle is verified in this case. In fact, from formula (45) and the well-known structure [L1] of finite-dimensional $\mathrm{Sl}_{2}$ modules, we can write

$$
\begin{aligned}
& P_{1}=\beta_{1}\left(\sum \frac{\partial^{2}}{\partial x^{2}}-\frac{\partial^{2}}{\partial t^{2}}\right)^{(p+1) / 2}\left(t^{2} d \mu\right) \\
& P_{2}=\beta_{2}\left(\sum \frac{\partial^{2}}{\partial x^{2}}-\frac{\partial^{2}}{\partial t^{2}}\right)^{(p-1) / 2}(t d \mu)
\end{aligned}
$$

where the $\beta_{i}$ are constants. Thus the propagator is computed explicitly and is by inspection supported on $C^{0}$.

The wave equation is of course one very special partial differential equation. Over the past 20 or so years, a major theme in the theory of partial differential equations has been the development of more or less explicit operational calculi to treat various general classes of equations. These calculi are of two main types, generally referred to as classes of pseudo-differential operators, and of Fourier integral operators. Since these theories grew in part out of abelian Fourier analysis and rely heavily on the Fourier transform, with hindsight we may not find it surprising that they may also be developed using the Heisenberg group. In fact some aspects of pseudo-differential operators, especially symbols, fit very naturally into the group-theoretic framework. For a detailed development of the discussion below, see [ $\mathrm{Hw} \mathrm{3}$; the ideas were also partially anticipated in [GLS], and indeed some go back to Weyl [Wy 1] and his original construction of the Heisenberg group.

The formula (21) tells us, as already noted, that $\delta(H, e)$ is taken by the representation $\rho$, in the Schrödinger model, to the algebra of all integral operators on $\mathcal{S}\left(\mathbf{R}^{n}\right)$ with kernels in $\mathcal{S}\left(\mathbf{R}^{2 n}\right)$. Since $\rho$ is also unitary, we can extend it to a linear isomorphism, still denoted $\rho$, from $\delta^{*}(H, e)$ to $\delta^{*}\left(R^{2 n}\right)$. The extended $\rho$ will still be an algebra isomorphism to the extent this makes sense. The Schwartz Kernel Theorem [T] identifies the space $\mathcal{S}^{*}\left(\mathbf{R}^{2 n}\right)$ to the space of all continuous maps from $\delta\left(\mathbf{R}^{n}\right)$ to $\delta *\left(\mathbf{R}^{n}\right)$. Hence any operator on $\mathcal{S}\left(\mathbf{R}^{n}\right)$, or even from $\delta\left(\mathbf{R}^{n}\right)$ to $\mathcal{S}^{*}\left(\mathbf{R}^{n}\right)$ may be viewed as coming, via $\rho$, from a distribution on $H$. In particular, pseudo-differential operators on $\mathcal{S}\left(\mathbf{R}^{n}\right)$ will 
arise in this way. Let us consider how the symbol of such a pseudo-differential operator looks from this viewpoint.

Actually we will not discuss the most common symbol, that of KohnNirenberg [KN], but a slightly different one, which happens to be the one written down by Weyl [Wy 1]. This symbol was also considered in [GLS], and used in the very general pseudo-differential calculus of [Hr]. We will call it the isotropic symbol. It differs in relatively minor ways from the Kohn-Nirenberg symbol, and explicit formulas relating the two can be found in [Hw 3] and [GLS].

The differences between the two symbols can be understood as follows. Functions in $\mathcal{S}(H, e)$ behave in a known way in the direction of the center $\mathbf{T}$ of $H$, so that to know $f$ in $\mathcal{S}(H, e)$ one needs only know it on a cross-section $C$ of the T-cosets in $H$. Thus by restriction one obtains an isomorphism from $\mathcal{S}(H, e)$ to some space of functions on $C$. By transport of structure one can define a convolution product on these functions, and one can map them to operators via $\rho$. If one coordinatizes $C$ by means of the projection of $H$ to $H / T \simeq \mathbf{R}^{2 n}$, then the convolution product on $\delta(H, e)$ transferred to $C$ looks like convolution on $\mathbf{R}^{2 n}$ "twisted" by the 2-cocycle defined by the cross-section $C$. For example, if one uses the cross-section $C_{1}=\{(y, x, 1)\}$ as in formula (20), then the convolution (denoted by \#) can be written

$$
f_{1} \# f_{2}\left(y^{\prime}, x^{\prime}\right)=\int_{\mathbf{R}^{2 n}} f_{1}(x, y) f_{2}\left(x^{\prime}-x, y^{\prime}-y\right) e^{2 \pi i x \cdot\left(y^{\prime}-y\right)} d x d y .
$$

On the other hand, if one uses the cross-section $C_{0}=e(W)$, where $e: \mathfrak{G} \rightarrow H$ is the exponential map and $W$ (and $\mathfrak{g}$ ) is as in (35), then one gets a slightly different convolution (denoted $\sharp$ ) and writable as

$$
f_{1} \boxminus f_{2}\left(w^{\prime}\right)=\int_{W} f_{1}(w) f_{2}\left(w^{\prime}-w\right) e^{-\pi i\left\langle w, w^{\prime}\right\rangle} d w .
$$

Here $\langle$,$\rangle is the symplectic form on W$ (cf. formula (35) et infra). Other cross-sections give still different convolution product formulas.

The cross-sections $C_{1}$ and $C_{0}$ have some particular virtues which make them more useful than other cross-sections, and for most purposes they are the only cross-sections one need consider. (In the theory of $\theta$-functions, another cross-section, not exactly of $H$, but of $H$ extended by the real scalar operators, plays a role.) The cross-section $C_{1}$ is most closely related to the conventional manner of writing differential operators, with the differentiations coming first, followed by the multiplications. The cross-section $C_{0}$, which we call the isotropic cross-section treats differentiations and multiplications in symmetrical fashion. Its virtue is that it is invariant under the full symplectic group $\operatorname{Sp}(W,\langle\rangle)=,\mathrm{Sp}$, and it is the only such cross-section. The cross-section $C_{1}$ is invariant only under the subgroup $\alpha\left(G L_{n}\right)$ of $\mathrm{Sp}$ (as defined in (32)).

With each cross-section we may associate a symbol map. The symbol associated to $C_{1}$ is the Kohn-Nirenberg symbol, which was built to conform with the traditional "derivatives first" manner of writing differential operators. The isotropic symbol which we will discuss below is the symbol associa- 
ted to the isotropic section $C_{0}$, and so is more natural in the group-theoretical framework.

The isotropic symbol is defined via the symplectic Fourier transform on $W$. For $f \in \mathcal{S}(W)$ write

$$
\hat{f}\left(w^{\prime}\right)=2^{-n} \int_{W} f(w) e^{-\pi i\left\langle w, w^{\prime}\right\rangle} d w .
$$

Our normalization of $d w$ makes $\frown$ unitary. Since it is defined via a symplectic rather than, as is usual, a symmetric form, the map $\cap$ has order 2 rather than order 4; that is,

$$
\widehat{f}=f .
$$

Comparing formulas (62) and (63) we note the intriguing fact that $\frown$ amounts to convolution on the right by the constant function $2^{-n}$. Furthermore, the function $2^{-n}$ on $W$ turns out to correspond via $\rho$ to the operator $\omega(-1)$, the image under the oscillator representation of minus the identity in $\mathrm{Sp}$. Finally, we note that of course $\cap$ can be extended to $\delta^{*}(W)$ by continuity. The extended $\frown$ is then the isotropic symbol, and will be denoted also by $\sigma$. Thus we have

$$
\sigma(D)=\overparen{D}=D \text { घ } 2^{-n}, \quad \rho(\sigma(D))=\rho(D) \omega(-1) .
$$

This symbol has, as it should, a symbolic calculus. To write it we require the usual notation. Set up coordinates in $W$ via the basis of formulas (11) and (12). Let $\alpha=\left(\alpha_{1}, \ldots, \alpha_{n}\right)$ denote a multi-index of nonnegative integers. We associate various quantities with $\alpha$ :

$$
|\alpha|=\sum_{i=1}^{n}\left|\alpha_{i}\right|, \quad \alpha !=\prod_{i=1}^{n} \alpha_{i} !, \quad \partial_{x}^{\alpha}=\prod_{i=1}^{n} \frac{\partial^{\alpha_{i}}}{\partial x_{i}^{\alpha_{i}}} .
$$

From (65) we see that, given say two distributions $D$ and $E$,

$$
\sigma(D \nvdash E)=D \text { } \sigma(E) \text {. }
$$

From (67) it is straightforward to compute that

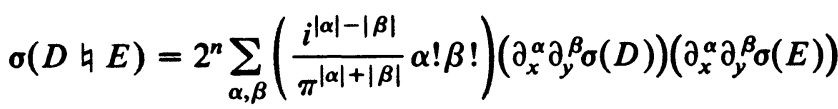

$$
\begin{aligned}
& =2^{n}\left(\sigma(D) \sigma(E)+\frac{i}{\pi}\{\sigma(D), \sigma(E)\}+\cdots\right) .
\end{aligned}
$$

Ignoring factors of $2^{n}, \pi$, etc., which are essentially artifacts of our choices of normalization, we see that the leading term of $\sigma(D \boxminus E)$ is just the pointwise product of $\sigma(D)$ and $\sigma(E)$, as it should be. The second term is the Poisson bracket of $\sigma(D)$ and $\sigma(E)$, and the higher terms are transvectants, certain classical differential invariants of the symplectic group.

An important use of the symbol is to estimate the size of the corresponding operator. A work horse estimate [Hr], [B] which can be proved very naturally in this group-theoretical context is the Calderón-Vaillancourt $(0,0)$ estimate [CV]. 
TheOREM (CAlderón-VaillanCourT). If $\sigma(D)$ and all its derivatives are bounded, then $\rho(D)$ is a bounded operator, with operator norm estimable in terms of the supremum norms on the derivatives.

This is actually a consequence of a general estimate for representations of locally compact groups, an estimate simple enough to derive. Let $G$ be a locally compact group, and $\sigma$ a representation of $G$ on a Hilbert space $\mathcal{H}$. Let $\mathcal{H C} . \mathcal{S}$. be the Hilbert-Schmidt operators on $\mathcal{H}$. On $\mathcal{H} . \mathcal{S}$. is defined the unitary representation $\sigma \otimes \sigma^{*}$ of $G$ by the rule

$$
\left(\sigma \otimes \sigma^{*}\right)(g)(T)=\sigma(g) T \sigma(g)^{-1}, \quad T \in \mathcal{H} . \mathcal{S} ., g \in G .
$$

Let $u, v$ be vectors in $\mathcal{H}$, and define the dyad $E_{u, v}$ in $\mathcal{H}$.S. by

$$
E_{u, v}(w)=(w, v) u, \quad w \in \mathcal{K}
$$

where $($,$) is the inner product in \mathcal{H}$.

One computes easily that

$$
\begin{aligned}
& E_{u, v} E_{u^{\prime}, v^{\prime}}=\left(u^{\prime}, v\right) E_{u, v^{\prime}} \\
& \sigma(g) E_{u, v}=E_{\sigma(g) u, v}, \quad E_{u, v} \sigma(g)^{-1}=E_{u, \sigma(g) v^{*}}
\end{aligned}
$$

If $v$ is a unit vector, then the map $\alpha: \mathcal{H} \rightarrow \mathcal{H} . \mathcal{S}$. defined by

$$
\alpha(u)=E_{u, v}
$$

is an isometric embedding, and from (71) we see that

$$
\alpha(\sigma(g) u)=\sigma(g) \alpha(u) \quad \alpha(u) \sigma(g)^{-1} E_{v, v}=\varphi(g) \alpha(u)
$$

where

$$
\varphi(g)=(v, \sigma(g) v)
$$

is the matrix coefficient of $\sigma$ defined by $v$. Combining the parts of (73) gives, assuming $\varphi(g) \neq 0$,

$$
\alpha(\sigma(g)(u))=\varphi(g)^{-1}\left(\sigma \otimes \sigma^{*}\right)(\alpha(u)) E_{v, v} .
$$

Thus if $f \in L^{1}(G)$ is supported away from the zero set of $\varphi$, we may integrate both sides of (75) to obtain

$$
\alpha\left(\left(\int_{G} f(g) \sigma(g) d g\right)(u)\right)=\left(\left(\int_{G} f(g) \varphi(g)^{-1}\left(\sigma \otimes \sigma^{*}\right)(g) d g\right)(\alpha(u))\right) E_{v, v}
$$

or more briefly

$$
\alpha(\sigma(f)(u))=\left(\sigma \otimes \sigma^{*}\right)\left(f \varphi^{-1}\right)(\alpha(u)) E_{v, v^{*}}
$$

Since $\alpha$ is an isometry and right multiplication by $E_{v, v}$ on $\mathcal{H} . \mathcal{S}$. has norm one, from (77) we get the estimate

$$
\|\sigma(f)\| \leqslant\left\|\sigma \otimes \sigma^{*}\left(f \varphi^{-1}\right)\right\|
$$

where $\|A\|$ denotes the operator norm of $A$.

REMARK. Formula (77) reminds one of the theory of Toeplitz operators [D], [V]. One might say it exhibits $\sigma(f)$ as a Toeplitz operator constructed from $\sigma \otimes \sigma^{*}\left(f \varphi^{-1}\right)$. 
Consider now the case when $G=H$, the Heisenberg group, and $\sigma=\rho$, the standard irreducible representation of $H$. Since the center $\mathrm{T}$ of $H$ is sent by $\rho$ to scalar operators, the representation $\rho \otimes \rho^{*}$ of $H$ will in fact factor to the abelian group $H / T$. Since representations of abelian groups are completely analyzable by means of the Fourier transform, and since the isotropic symbol amounts essentially to Fourier transform, it is perhaps plausible that (78) for $H$ and $\rho$ would imply a result like the $(0,0)$ estimate. At any rate, it does.

Although the isotropic symbol does what it should and its definition (65) is clean, it may still seem less than completely natural. As a final point, we will attempt to further motivate (65) by showing it is consistent with the Kirillov orbit theory for nilpotent groups, as specialized to $H$. Such a motivation is entirely unhistorical, but it may suggest possibilities for future research.

Let $\tilde{H}$ be the universal covering group of $H$. The group $\tilde{H}$ has the same Lie algebra $\mathscr{Q}$ as does $H$, and the exponential map

$$
\tilde{e}: \$ \rightarrow \tilde{H}
$$

is now a diffeomorphism. Let $\mathscr{Z}$ denote the center of $\mathscr{G}$ and $Z=\exp \mathscr{Z}$ the center of $\tilde{\boldsymbol{H}}$. Above in equation (35) we have identified $\mathscr{Z}$ with $\mathbf{R}$. We will identify $Z$ to $\mathbf{R}$ also in such a way that exp: $\mathscr{Z} \rightarrow Z$ is the identity map on $\mathbf{R}$. Then we may write

$$
\tilde{H}=\{(\exp w, t): w \in W, t \in \mathbf{R}\}
$$

with $W$ also as in (35). The group law in $\tilde{H}$ then reads

$$
\left(\exp w_{1}, t_{1}\right)\left(\exp w_{2}, t_{2}\right)=\left(\exp \left(w_{1}+w_{2}\right), t_{1}+t_{2}+\frac{1}{2}\left\langle w_{1}, w_{2}\right\rangle\right)
$$

According to Kirillov [Kr], the irreducible unitary representations of $\tilde{\boldsymbol{H}}$ are in natural bijection with the orbits of $\tilde{H}$ (or $H$, since the center acts trivially) in $\mathscr{S}^{*}$, the dual of $\mathscr{B}$, under the co-adjoint action. In accordance with the Stone-von Neumann Theorem, the orbits which are not single points are hyperplanes of the form $\lambda+\mathscr{Z}^{\perp}$, where $\mathscr{Z}^{\perp}$ is the annihilator in $\mathscr{S}^{*}$ of $\mathscr{Z}$ and $\lambda$ is some element in the orbit. An irreducible representation $\tau$ of $\tilde{H}$ determines a central character $\chi_{r}$ on $Z$, and is determined by $\chi_{r}$ if this is nontrivial. If $\tau$ corresponds to the orbit $\lambda+\mathscr{Z}^{\perp}$, then

$$
e^{2 \pi i \lambda(z)}=\chi_{r}(\exp z), \quad z \in \mathscr{Z} .
$$

There will be a unique point $\lambda_{0}$ in $\left(\lambda+\mathscr{L}^{\perp}\right) \cap W^{\perp}$. Thus having a cross-section $W$ to $\mathscr{Z}$ is equivalent to having a base point $\lambda_{0}$ in $\lambda+\mathscr{I}^{\perp}=\lambda_{0}$ $+\mathscr{Z}^{\perp}$. From now on we will take $\lambda=\lambda_{0}$. Note that $\mathcal{Z}^{\perp}$ is naturally dual to $W$. On the other hand $W$ is self-dual by virtue of the symplectic form $\lambda([]$, defined on it. Therefore we have an isomorphism

$$
\beta: W \rightarrow \mathbb{Z}^{\perp}
$$

defined by

$$
\beta\left(w^{\prime}\right)(w)=\lambda\left(\left[w^{\prime}, w\right]\right)
$$

We can define in a natural manner a Fourier transform ^ from $\mathcal{S}(\xi)$ to $\mathcal{S}\left(\mathfrak{B}^{*}\right)$ by

$$
\hat{f}(\mu)=\int_{\mathscr{Q}} f(x) e^{2 \pi i \mu(x)} d x, \quad f \in \mathcal{S}(\mathfrak{B}), \mu \in \mathscr{Q}^{*}
$$


Here $d x=d w d z$ where $d w$ is self-dual Haar measure on $W$ and $d z$ is Lebesgue measure on $\mathscr{Z} \simeq \mathbf{R}$. We may extend (84) to tempered distributions by continuity.

If the representation $\tau$ corresponds to the plane $\lambda_{0}+\mathscr{Z}^{\perp}$ in $\mathfrak{S}^{*}$, then the analogue for $\tilde{H}$, with its noncompact center, of the space $\delta(H, e)$ is the space $\delta(\tilde{H}, \tau)$ of functions $f$ on $\tilde{H}$ such that

$$
L_{\exp z}(f)=e^{2 \pi i \lambda_{0}(z)} f, \quad z \in \mathscr{Z},(f \circ \exp ) \mid W \in \delta(W) .
$$

If, under the identification of (35) of $\mathscr{Z}$ with $\mathbf{R}$, we have $\lambda_{0}(z)=z$, then $\tau$ is simply the lift to $\tilde{H}$ of the representation $\rho$ of $H$, and the space $\delta(\tilde{H}, \tau)$ is simply the pullback to $\tilde{H}$ of $\delta(H, e)$. Fix this $\tau$. Then for $f$ in $\delta(\tilde{H}, \tau)$ we may speak of the isotropic symbol $\sigma(f)$ as a function on $W$. On the other hand $f$ may be regarded as a distribution on $H$, and its pullback to $\$$ via exp is a distribution on $\mathfrak{Q}$. Thus we may consider

$$
(f \circ \exp )^{-}
$$

which will be a distribution on $\mathfrak{S}^{*}$, and will have the form

$$
(f \circ \exp )^{\wedge}=\tilde{f} d v
$$

where $\tilde{f}$ is a smooth function on $\lambda_{0}+\mathscr{Z}^{\perp}$ and $d \nu$ is an appropriately normalized Lebesgue measure on $\lambda_{0}+\mathscr{Z}^{\perp}$. Comparing (84) and (86) with the definition (63) of the isotropic symbol, and using (83) we see that

$$
\sigma(f)(w)=2^{-n \tilde{f}\left(\lambda_{0}+\beta(w / 2)\right)} .
$$

Again, the factors of 2 here are essentially irrelevant.

Formula (87) tells us that the symbol as it is understood in P.D.E. is consistent with the map

$$
f \rightarrow(f \circ \exp )^{\wedge}
$$

from $\delta(H)$ to $\delta\left(\mathfrak{S}^{*}\right)$. But the map (88) has been a basic part of harmonic analysis on nilpotent groups since Kirillov's original paper [Kr] where it was used to construct the characters of irreducible representations. This suggests that the map (88) might be usefully construed as a symbol map for a general nilpotent group. It might be used, for example, in deriving norm estimates for the images of functions under representations of the group, including the regular representation. There has developed over the past few years a theory of "singular integral operators" on nilpotent groups with dilations, but this theory has used nilpotent harmonic analysis in a minimal way. The motivating example for this theory was again provided by the Heisenberg group, and appeared in a paper of Knapp and Stein [KS]. Because of the dilation-invariance of the Knapp-Stein operators, formula (87) implies that the Knapp-Stein result follows from the Calderón-Vaillancourt $(0,0)$ estimate. On the other hand, it is a simple matter to dilate the Calderón-Vaillancourt (C-V) result. We will do this explicitly because it further illuminates the group-theoretic basis of this estimate and because it suggests generalization.

Since $H$ acts on $\oiint^{*}$ via the co-adjoint action $\mathrm{Ad}^{*}$, by applying $\mathrm{Ad}^{*}$ to $\$$ we have a Lie algebra of vector fields on $\mathfrak{S}^{*}$. Let $\left\{X_{j}, Y_{j}, T\right\}$ be the basis for $\$$ defined in (11), and let $\left\{X_{j}^{*}, Y_{j}^{*}, T^{*}\right\}$ be the dual basis for $\mathfrak{S}^{*}$. Let $x_{j}^{*}, y_{j}^{*}, z^{*}$ be the coordinates on $\mathfrak{S}^{*}$ with respect to this dual basis. Then 


$$
\operatorname{Ad}^{*}\left(X_{j}\right)=z^{*} \frac{\partial}{\partial y_{j}^{*}}, \quad \operatorname{Ad}^{*}\left(Y_{j}\right)=-z^{*} \frac{\partial}{\partial x_{j}^{*}}, \quad \operatorname{Ad}^{*}(T)=0 .
$$

Thus, restricted to any hyperplane $\lambda_{0}+\mathscr{Z}^{\perp}$, the vector fields $\operatorname{Ad}^{*}(W)$ are just the constant coefficient vector fields, i.e., just give first order partial derivatives, and it is in terms of the symbol and its derivatives that the C-V estimates is couched. Thus the formula (89) together with an inspection of how the constants in C-V depend on Planck's constant allow one to derive results like this.

THEOREM. Suppose $D \in \mathcal{S}^{*}(\tilde{H})$ is such that $(D \circ \exp )^{\wedge}$ is a smooth function and the functions obtained by applying any product of the operators $z^{*-1 / 2} \operatorname{Ad}^{*}(w), w \in W$, are bounded. Then for any unitary representation $\tau$ of $\tilde{H}$, the operator $\tau(D)$ is bounded. The bound is independent of $\tau$ and depends only on the $\infty$-norms of the functions described above. In particular, left convolution by $\tau$ is bounded.

It is not difficult to extend a result like this to a general 2-step nilpotent group. Then formula (78) would permit use of the 2-step result to get estimates for irreducible representations of 3-step nilpotent groups. Thus one might envisage a bootstrap argument yielding analogues of $\mathrm{C}-\mathrm{V}$ for general nilpotent groups.

To conclude, this paper has tried to illustrate how it can be helpful and pleasing to consider various basic or classical topics in harmonic analysis in terms of the Heisenberg group. It does not begin to exhaust the list of possible topics, even in this relatively restricted domain of discourse. The topics chosen reflect the author's predilections and limitations more than the bounds of known applicability of the Heisenberg group to analysis. In fact, the article of Stein which framed the first part of this article ends with a discussion of some applications of the Heisenberg group, and Stein's work has often involved $\boldsymbol{H}$. And it should also be clear that the perspective propagandized here has many possibilities for future development.

I would like to thank the University of Maryland and Ron Lipsman, whose invitation to deliver lectures for their Special Year in Harmonic Analysis provided the occasion for writing a preliminary version of this article.

\section{REFERENCES}

[B] R. Beals, A general calculus of pseudodifferential operators, Duke Math. J. 42 (1975), 1-42.

[CV] A. Calderón and R. Vaillancourt, On the boundedness of pseudo-differential operators, J. Math. Soc. Japan 23 (1971), 374-378.

[Cr] P. Cartier, Quantum mechanical commutation relations and theta functions, Proc. Sympos. Pure Math., vol. 9, Amer. Math., Soc., Providence, R. I., 1966, pp. 361-383.

[D] R. G. Douglas, Banach algebra techniques in operator theory, Academic Press, New York, 1972.

[GLS] A. Grossman, G. Loupias and E. Stein, An algebra of pseudo-differential operators and quantum mechanics in phase space, Ann. Inst. Fourier (Grenoble) 18 (1968), 343-368.

[Hr] L. Hörmander, The Weyl calculus of pseudodifferential operators, Comm. Pure Appl. Math. 32 (1979), 359-443.

[Hw1] R. Howe, On some results of Strichartz and of Rallis and Schiffmann, J. Functional Anal. 32 (1979), 297-303. 
[Hw2] ___ Remarks on Huyghens' principle (preprint).

[Hw3] __ Quantum mechanics and partial differential equations, J. Functional Anal. (to appear).

[Kr] A. A. Kirillov, Unitary representations of nilpotent Lie groups, Uspehi Mat. Nauk. 17 (1962), 57-110; Russian Math. Surveys 17 (1962), 53-104.

[KS] A. Knapp and E. Stein, Intertwining operators for semisimple groups, Ann. of Math. (2) 93 (1971), 489-578.

[KN] J. Kohn and L. Nirenberg, An algebra of pseudodifferential operators, Comm. Pure Appl. Math. 18 (1965), 269-305.

[L1] S. Lang, $S L_{2}(R)$, Addison-Wesley, Reading, Mass., 1975.

[L2] __ Real analysis, Addison-Wesley, Reading, Mass., 1969.

[Ma] G. Mackey, Unitary representations of group extensions. I, Acta Math. 99 (1958), 265-311.

[MW] C. Moore and J. Wolf, Square integrable representations of nilpotent groups, Trans. Amer. Math. Soc. 185 (1973), 445-462.

[Mm] D. Mumford, On the equations defining abelian varieties. I, II, III, Invent. Math. 2 and 3, (1966) and (1967).

[RS] S. Rallis and G. Schiffmann, Weil representation. I. Intertwining distributions and discrete spectrum, Mem. Amer. Math. Soc. No. 231 (1980).

[Sg] I. Segal, Transforms for operators and symplectic automorphisms over a locally compact abelian group, Math. Scand. 13 (1963), 31-43.

[Sh] D. Shale, Linear symmetries of free boson fields, Trans. Amer. Math. Soc. 103 (1962), 149-167.

[St] R. Strichartz, Fourier transforms and non-compact rotation groups, Indiana Univ. Math. J. 24 (1974), 499-526.

[T] F. Treves, Topological vector spaces, distributions and kernels, Academic Press, New York, 1967.

[V] U. Venugopalkrishna, Fredholm operators associated with strongly pseudoconvex domains in $C^{n}$, J. Functional Anal. 9 (1972), 349-372.

[vN]. J. von Neumann, Die eindeutigkeit der Schröderschen Operatoren, Math. Ann. 104 (1931), 570-578.

[Wi] A. Weil, Sur certains groupes d'opérateurs unitaires, Acta. Math. 111 (1964), 145-211.

[Wy1] H. Weyl, The theory of groups and quantum mechanics, Methuen, London, 1931.

[Wy2] __ The classical groups, Princeton Univ. Press, Princeton, N. J., 1939.

Department of Mathematics, Yale University, New Haven, Connecticut 06520 
